\title{
Weapon Operational Effectiveness Evaluation Credibility Analysis Based on Fuzzy AHP Colony
}

\author{
Guo Rui ${ }^{1, a, *}$, Dou JiHua ${ }^{2}$ \\ 1,2667\#, Jiefang road, Zhongshan District, Dalian City, Liaoning Province, 116018 \\ a msegr@163.com \\ *corresponding author
}

Keywords: Ship-to-air missile; system operational effectiveness; evaluation credibility; fuzzy AHP; colony

\begin{abstract}
Weapon operational effectiveness approach is proposed, the missile system operational effectiveness evaluation credibility index system is proposed, the missile system operational effectiveness evaluation credibility analysis method based on fuzzy AHP colony is proposed. The missile system operational effectiveness evaluation credibility index weight, through introducing triangle fuzzy number, can be obtained by using fuzzy AHP colony method. Single expert weight calculation method of colony decision-making is proposed, the missile system operational effectiveness evaluation credibility value can be obtained. The proposed methods can provide the research idea for other system operational effectiveness evaluation credibility analysis.
\end{abstract}

\section{Introduction}

Weapon operational effectiveness evaluation credibility is of fuzzy and qualitative concept, the missile system operational effectiveness evaluation credibility index system is established, the missile system operational effectiveness evaluation credibility is analyzed.

\section{Weapon operational effectiveness evaluation process}

Weapon operational effectiveness evaluation process is as follows.

Evaluation step one is to the specific combat scenario of the missile system, the contents of the specific combat scenario includes combat posture and character, formation intention, combat environment, the missile system constitution and structure, the missile system air defense mission and so on.

Evaluation step two is to select the missile system operational effectiveness evaluation methodology.

Evaluation step three is to establish the missile system operational effectiveness evaluation model. 
Evaluation step four is to get the initial data required by the missile system operational effectiveness evaluation.

Evaluation step five is to estimate the numerical values of the missile system operational effectiveness evaluation method parameters.

Evaluation step six is the missile system operational effectiveness evaluation through the model.

Evaluation step seven is the missile system operational effectiveness evaluation credibility validation.

\section{Weapon operational effectiveness evaluation credibility index system}

Weapon operational effectiveness evaluation credibility index system is shown in Fig.1. The missile system operational effectiveness evaluation credibility factor is defined by $U$, the air defense plan verification factor is defined by $U_{1}$, the air defense plan integrity verification factor is defined by $U_{11}$, the air defense plan initial data veracity verification factor is defined by $U_{12}$, the missile system operational effectiveness evaluation method verification factor is defined by $\mathrm{U}_{2}$, the missile system operational effectiveness evaluation method rationality verification factor is defined by $U_{21}$, the missile system operational effectiveness evaluation method logicality verification factor is defined by $U_{22}$, the missile system operational effectiveness evaluation model verification factor is defined by $U_{3}$, the missile system operational effectiveness evaluation model type verification factor is defined by $U_{31}$, the missile system operational effectiveness evaluation model establishment method verification factor is defined by $U_{32}$, the missile system operational effectiveness evaluation model validation factor is defined by $U_{4}$, the missile system operational effectiveness evaluation model type validation factor is defined by $U_{41}$, the missile system operational effectiveness evaluation model establishment method validation factor is defined by $U_{\mathbf{4 2}}$, the missile system operational effectiveness evaluation result validation factor is defined by $U_{5}$, the missile system operational effectiveness evaluation result figure method validation factor is defined by $U_{51}$, the missile system operational effectiveness evaluation result simulation method validation factor is defined by $U_{\mathbf{5 2}}$, the missile system operational effectiveness evaluation result experimentation method validation factor is defined by $U_{\mathbf{5 3}}$, the missile system operational effectiveness evaluation validation factor is defined by $\mathrm{U}_{6}$.

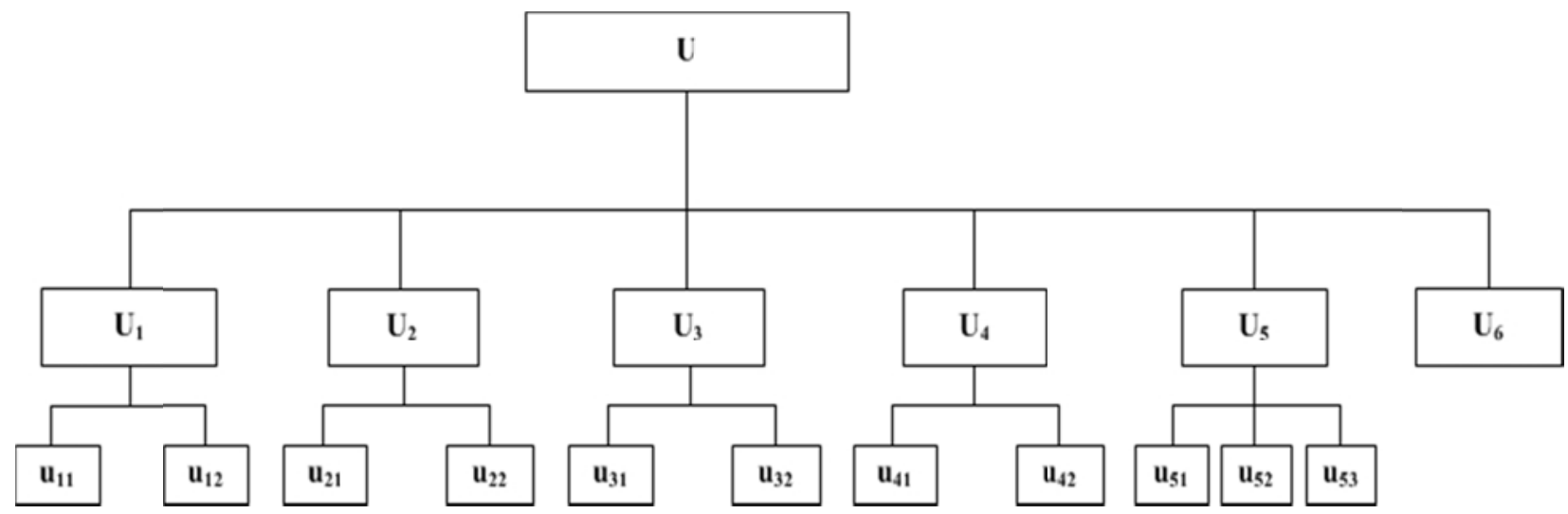

Figure 1 Weapon operational effectiveness evaluation credibility index system 


\section{Weapon operational effectiveness evaluation credibility analysis based on fuzzy AHP colony decision-making}

Assume that expert aggregation of fuzzy AHP colony decision-making is defined by $Z=\left\{z_{1}, z_{2}, \ldots, z_{p}\right\}$. Based on Figure 1, assume that the number of sub-indexes for certain index (defined by $\mathrm{B}$ ) is $\mathrm{m}$, and the index evaluation aggregation is defined by $\mathrm{G}=\left\{b_{1}, b_{2}, \ldots, b_{m}\right\}$. Weapon operational effectiveness evaluation credibility analysis process based on fuzzy AHP colony decision-making is as follows.

\subsection{Single expert evaluation value calculation}

Because of calculation difficulty of weapon operational effectiveness evaluation credibility data, the index B is evaluated by single expert (defined by $z_{l}$ ), through fuzzy mathematical theory, single index can be fuzzily evaluated, the missile system operational effectiveness can be evaluated by the expert through fuzzy comprehensive evaluation.

\subsubsection{Fuzzy comprehensive evaluation of single index}

Assume that evaluation aggregation is defined by $\mathrm{V}=\left\{\mathrm{V}_{1}, \mathrm{~V}_{2}, \mathrm{~V}_{3}, \mathrm{~V}_{4}, \mathrm{~V}_{5}\right\}=\{$ very good, good, common, bad, very bad\}.

Assume that the subjection value of the index (defined by $b_{i}$ ) to $\mathrm{V}$ by the expert $z_{l}$ is $r_{\text {lia }}$, and the fuzzy relation vector is defined by $\mathrm{R}_{1}, 1 \leq \mathrm{i} \leq \mathrm{m}, 0 \leq r_{\text {lia }} \leq 1,1 \leq \mathrm{a} \leq 5, \mathrm{R}_{1}=\left(r_{l i 1}, r_{l i 2}, r_{l i 3}, r_{l i 4}, r_{l i 5}\right)$.

\subsubsection{Index weight calculation}

Through triangle fuzzy number, fuzzy estimation matrix is established, the index weight can be calculated.

Assume that triangle fuzzy number mutual supplement estimation matrix is $\tilde{P}_{l}$, and triangle fuzzy number is $\tilde{p}_{l i j}=\left(a_{l i j}, b_{l i j}, c_{l i j}\right), 0 \leqslant a_{l i j} \leqslant b_{l i j} \leqslant c_{l i j} \leqslant 1, \quad 1 \leqslant \mathrm{j} \leqslant \mathrm{m}, \quad 1=1,2, \ldots, \mathrm{p}, \tilde{P}_{l}$ can be defined by $\tilde{P}_{l}=\left(\tilde{p}_{l i j}\right)_{m \times m}$. When $\mathrm{i}=\mathrm{j}, a_{l i j}=b_{l i j}=c_{l i j}=0.5$. when $\mathrm{i} \neq \mathrm{j}, a_{l i j}+c_{l j i}=1, b_{l i j}+b_{l j i}=1, c_{l i j}+a_{l j i}=1$.

When the index $b_{i}$ and index $b_{j}$ are compared by significance, the linguistic variables with their corresponding triangle fuzzy numbers are shown in Table 1.

Table 1 Linguistic variables with their corresponding triangle fuzzy numbers

\begin{tabular}{|c|c|}
\hline $\begin{array}{c}\text { Index significance } \\
\text { comparison }\end{array}$ & $\begin{array}{c}\text { corresponding triangle fuzzy } \\
\text { number }\end{array}$ \\
\hline $\mathrm{b}_{\mathrm{j}}$ is more important than $\mathrm{b}_{\mathrm{i}}$ & $(0,0.1,0.3)$ \\
\hline $\mathrm{b}_{\mathrm{j}}$ is important than $\mathrm{b}_{\mathrm{i}}$ & $(0.1,0.3,0.5)$ \\
\hline $\mathrm{b}_{\mathrm{i}}$ is the same important as & $(0.3,0.5,0.7)$ \\
\hline $\mathrm{b}_{\mathrm{j}}$ & $(0.5,0.7,0.9)$ \\
\hline $\mathrm{b}_{\mathrm{i}}$ is more important than $\mathrm{b}_{\mathrm{j}}$ & $(0.7,0.9,1.0)$ \\
\hline $\mathrm{b}_{\mathrm{i}}$ is important than $\mathrm{b}_{\mathrm{j}}$ & \\
\hline
\end{tabular}


Assume that fuzzy comprehensive evaluation value of the index $b_{i}$ by the expert $z_{l}$ is $\tilde{u}_{l i}$, based on the triangle fuzzy number mutual supplement estimation matrix $\tilde{P}_{l}, \tilde{u}_{l i}=\left(d_{l i}, e_{l i}, f_{l i}\right)=$

$$
\left(\sum_{j=1}^{m} a_{l i j}, \sum_{j=1}^{m} b_{l i j}, \sum_{j=1}^{m} c_{l i j}\right) \otimes\left(\sum_{i=1}^{m} \sum_{j=1}^{m} a_{l i j}, \sum_{i=1}^{m} \sum_{j=1}^{m} b_{l i j}, \sum_{i=1}^{m} \sum_{j=1}^{m} c_{l i j}\right)^{-1} \approx\left(\frac{\sum_{j=1}^{m} a_{l i j}}{\sum_{i=1}^{m} \sum_{j=1}^{m} c_{l i j}}, \frac{\sum_{j=1}^{m} b_{l i j}}{\sum_{i=1}^{m} \sum_{j=1}^{m} b_{l i j}}, \frac{\sum_{j=1}^{m} c_{l i j}}{\sum_{i=1}^{m} \sum_{j=1}^{m} a_{l i j}}\right) .
$$

Assume that left expectation value of the fuzzy comprehensive evaluation value $\tilde{u}_{l i}$ is $Q_{L}\left(\tilde{u}_{l i}\right)$, and the right expectation value of the fuzzy comprehensive evaluation value $\tilde{u}_{l i}$ is $Q_{R}\left(\tilde{u}_{l i}\right) . Q_{L}\left(\tilde{u}_{l i}\right)$ $=\left(d_{l i}+e_{l i}\right) / 2, Q_{R}\left(\tilde{u}_{l i}\right)=\left(e_{l i}+f_{l i}\right) / 2$.

Assume that that expectation value of the fuzzy comprehensive evaluation value $\tilde{u}_{l i}$ is $Q\left(\tilde{u}_{l i}\right)$, $Q\left(\tilde{u}_{l i}\right)=\mu Q_{L}\left(\tilde{u}_{l i}\right)+(1-\mu) Q_{R}\left(\tilde{u}_{l i}\right)$, where $\mu$ is optimistic and pessimistic coefficient, $0<\mu<1$. When $\mu>0.5$, which indicates that the expert $z_{l}$ is pessimistic. When $\mu=0.5$, which indicates that the expert $z_{l}$ is neutral. When $\mu<0.5$, which indicates that the expert $z_{l}$ is optimistic.

Assume that the weight of the index $b_{i}$ given by the expert $z_{l}$ is $w_{l i}, w_{l i}=Q\left(\tilde{u}_{l i}\right) / \sum_{i=1}^{m} Q\left(\tilde{u}_{l i}\right)$. When $w_{l i}$ value is bigger, which indicates that the expert $z_{l}$ thinks that the index $b_{i}$ is more important.

\subsubsection{Single expert evaluation value calculation}

Assume that the evaluation value of the index $\mathrm{B}$ given by the expert $z_{l}$ is $\mathrm{H}_{1 \mathrm{~B}}, \mathrm{H}_{\mathrm{IB}}=\left(h_{I B 1}, h_{I B 2}, h_{I B 3}\right.$, $\left.h_{l B 4}, h_{l B 5}\right)=\left(\sum_{i=1}^{m} r_{l i 1} w_{l i}, \sum_{i=1}^{m} r_{l i 2} w_{l i}, \sum_{i=1}^{m} r_{l i 3} w_{l i}, \sum_{i=1}^{m} r_{l i 4} w_{l i}, \sum_{i=1}^{m} r_{l i 5} w_{l i}\right)$. Assume that the missile system operational effectiveness evaluation credibility value of the index $U$ given by the expert $z_{l}$ is $H_{l U}$, based on the index system in the Figure 1, according to the above method, $\mathrm{H}_{\mathrm{lU}}$ can be defined by

$$
\mathrm{H}_{\mathrm{IU}}=\left(h_{I U 1}, h_{I U 2}, h_{I U 3}, h_{I U 4}, h_{I U 5}\right)
$$

\subsection{Expert weight calculation}

Expert weight can be divided by former and latter weight. Former weight is related with the expert's overpassed information, including the influence factors such as specialty level, experience, information structure, familiarity degree of the operational effectiveness evaluation. Assume that the former weight for the expert $z_{l}$ is $\alpha_{l x}$, it can be calculated by hiberarchy analysis method, $0 \leqslant$ $\alpha_{l x} \leqslant 1, \quad \alpha_{1 x}+\alpha_{2 x}+\ldots+\alpha_{p x}=1$.

Assume that the latter weight for the expert $z_{l}$ is $\alpha_{l h}$, it can be calculated by

$$
\alpha_{l h}=\frac{\max \left\{h_{I U 1}, h_{I U 2}, h_{I U 3}, h_{I U 4}, h_{I U 5}\right\}}{\sum_{s=1}^{p} \max \left\{h_{s U 1}, h_{S U 2}, h_{S U 3}, h_{S U 4}, h_{s U 5}\right\}}
$$


where $0 \leqslant \alpha_{l h} \leqslant 1, \quad \alpha_{1 h}+\alpha_{2 h}+\ldots+\alpha_{p h}=1$.

The weight for the expert $z_{l}$ can be calculated by

$$
\alpha_{l}=\frac{\eta \alpha_{l x}+(1-\eta) \alpha_{l h}}{\sum_{w=1}^{p} \eta \alpha_{w x}+(1-\eta) \alpha_{w h}}
$$

where $0 \leqslant \alpha_{l} \leqslant 1, \quad \alpha_{1}+\alpha_{2}+\ldots+\alpha_{p}=1,0 \leqslant \eta \leqslant 1$.

\subsection{Expert colony evaluation value}

Through the expert weight value $\alpha_{l}$ and the evaluation value $\mathrm{H}_{1 \mathrm{U}}$ for the expert $z_{l}$, the expert colony evaluation value (defined by $\tilde{H}$ ) can be calculated by

$$
\tilde{H}=\left(h_{1}, h_{2}, h_{3}, h_{4}, h_{5}\right)=\left(\sum_{l=1}^{p} \alpha_{l} h_{l U 1}, \sum_{l=1}^{p} \alpha_{l} h_{I U 2}, \sum_{l=1}^{p} \alpha_{l} h_{I U 3}, \sum_{l=1}^{p} \alpha_{l} h_{I U 4}, \sum_{l=1}^{p} \alpha_{l} h_{I U S}\right)
$$

\subsection{Non-fuzziness}

Through the maximum value method, weapon operational effectiveness evaluation credibility value is the maximum value of the $\tilde{H}=\max \left\{h_{1}, h_{2}, h_{3}, h_{4}, h_{5}\right\}$.

\section{Conclusions}

Based on weapon operational effectiveness evaluation process, the missile system operational effectiveness evaluation credibility index system is proposed, the missile system operational effectiveness evaluation credibility analysis method based on fuzzy AHP colony decision-making is proposed. The proposed method is simple and practical, and the attempt to carry through the missile system operational effectiveness evaluation credibility analysis, and provide a new research methodology for other weapon system operational effectiveness evaluation credibility analysis.

\section{References}

[1] Kevin W.Brown,Measuring the Effectiveness of Weapons Systems in Terms of System Attributes,Master's thesis, Naval Postgraduate School, Monterey, CA,1995.

[2] Teng Kenan. Ship-to-air missile system operational effectiveness evaluation under saturation attack[J]. missile and guidance transaction, 2005, 25(3): 37-39.

[3] Dou Jihua. Ship-to-air missile system operational effectiveness evaluation simulation study[J]. missile and guidance transaction, 2007, 27(1): 290-293.

[4] Knut O.Flaathen,A Methodology to Find Overall System Effectiveness in a Multicriterion Environment Using Surface to Air Missile Weapon Systems as an Example,Master`s thesis, Naval Postgraduate School, Monterey, CA,September 1981.

[5] Li Changsheng, Jiang Jingshao, Zeng Xianzhao, Liu Yiyong. Military operations research method study and application[M]. Military Science Publishing House, 2006. 